\title{
Microbial community with the ability to biodegradation perchlorate in a bio-electrochemical reactor
}

\author{
Yu Zhong ${ }^{1}$, Li He ${ }^{2, *}$, Guangyi $\mathrm{Fu}^{1, *}$, Youze $\mathrm{Xu}^{1}$, Lixia Xie ${ }^{3}$, Yingxiang Cheng ${ }^{1}$, YuanYuan $\mathrm{Zhao}^{1}$, Yaqun Qiu ${ }^{1}$, Renjun \\ Xiang ${ }^{1}$, Xian $\mathrm{Gao}^{4}$ \\ ${ }^{1}$ Hunan Research Academy of Environmental Science, Hunan Provincial Key Laboratory of Water Pollution Control Technology, \\ Changsha 410004, China \\ ${ }^{2}$ College of Environmental Science and Engineering, Hunan University, Changsha 410082, China \\ ${ }^{3}$ College of Life Sciences and Chemistry, Hunan University of Technology, Zhuzhou 412007, P. R. China \\ ${ }^{4}$ Hunan Research Academy of Environmental Science Technology Consulting Co., Ltd, Changsha 410004, China
}

\begin{abstract}
In this study, the sediments from the Liuyang River (Hunan Province, China) were used as an inoculum to bio-reduction perchlorate in a bio-electrochemical reactor (BER). The efficient degradation of perchlorate was found in the BER by utilizing the hydrogen as electron donor. When the current intensity was $10 \mathrm{~mA}$ and HRT was $72 \mathrm{~h}$, the removal rate of perchlorate (initial concentration was $5 \mathrm{mg} / \mathrm{L}$ ) reached $84.13 \%$ and a removal flux of $178.68 \mathrm{mg} / \mathrm{m} 2 \cdot \mathrm{d}$ was achieved. High-throughput sequencing analysis confirmed that the biofilm in the reactor had been successfully acclimated, and the system could achieve perchlorate reduction effectively. Firmicutes and Bacteroidetes were the dominant phyla during inoculation phase, and Actinobacteriria, Proteobacteria, and Tenericutes also constituted a low proportion in the biofilm. Bacilli and Clostridia were dominant at class-level both in inoculum and biofilm, with the relative abundance about $56 \%-72 \%$ and $17 \%-23 \%$, respectively. These results confirmed that the biofilm in the BER system had been successfully formed, and the BER system could biodegradation perchlorate effectively.
\end{abstract}

\section{Introduction}

The perchlorate contamination has received great attention over the past years. Perchlorate is widely distributed in the natural environment, not only because of the widespread use of explosives and disinfectants [1,2], but also attribute to its inherent properties, such as high solubility, slow response, and low adsorption [3, 4]. In general, the concentration of perchlorate in groundwater is below $100 \mu \mathrm{g} / \mathrm{L}$, but it can reach $20 \mathrm{mg} / \mathrm{L}$ or higher level in some surface waters $[5,6]$. Perchlorate can threat human health by interfering with the production of thyroid hormones, and subsequently inhibiting the development of the skeletal system and the central nervous system of infant [7]. Owing to the perchlorate charge and ionic radius are very close to that of iodide, perchlorate could compete with iodide for entry into the thyroid gland, leading to inhibition of iodine uptake and further affecting thyroid function [8].Membrane filtration, ion exchange (IX), and electrochemical reduction are typically strategies for removing perchlorate from water $[2,9,10]$. However, it has been pointed out that these methods are limited in terms of perchlorate removal [11]. Biological reduction has been proven to be the most effective and economically attractive approach for treating perchloratecontaminated waters $[11,12]$. It could completely reduce perchlorate by microorganisms, perchlorate-reducing bacteria (PRB) [13], under anaerobic conditions in the presence of a suitable electron donor according to following reaction: $\mathrm{ClO}_{4}{ }^{-} \rightarrow \mathrm{ClO}_{3}{ }^{-} \rightarrow \mathrm{ClO}_{2}{ }^{-} \rightarrow \mathrm{Cl}^{-}+\mathrm{O}_{2} \quad[11$, 12]. Electron donor could be organic substrate, e.g. acetate, ethanol, and inorganic electron donor such as $\mathrm{H}_{2}$. As a clean and non-toxic energy, $\mathrm{H}_{2}$ has been used as an electron donor for microbial reduction of perchlorate in many studies $[2,14,15]$. However, the explosive risk of $\mathrm{H}_{2}$ in transportation, storage and utilization limits its extensive use. In addition, some reports showed that the internal (electrochemical or chemical) production of $\mathrm{H}_{2}$ could be more effectively utilized by bacteria than that of external supplied [16].

In this research, a bio-electrochemical reactor (BER) was developed to remove perchlorate by using $\mathrm{H}_{2}$ as electron donor. In BER, $\mathrm{H}_{2}$ is generated on site by electrolysis of water and completely utilized by the bacteria immobilized on the cathode surface $[16,17]$. In addition, the high-throughput sequencing technology was used to reveal the relationship between microbial community structure and perchlorate removal performance in bio-electrochemical reactor.

\section{Experimental Methods}

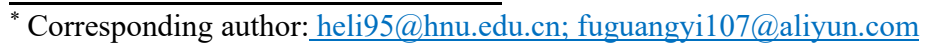




\subsection{Reactor configuration}

The reactor system mainly consisted of two cylindrical polyethylene glasses, an anode compartment and a cathode compartment (Fig. 1). A DC regulated power was connected externally to provide power for the reactor. Titanium rods were provided as anode and cathode in the anode compartment and the cathode compartment, respectively. The activated carbon felt was fixed around the cathode. A peristaltic pump and water tank were provided for continuous water ingress. A magnetic stirrer was used to mix the liquid of the compartment. The liquid volume was $0.98 \mathrm{~L}$ in cathode compartment. A proton exchange membrane (Nafion 117, Dupont, USA) separated two compartments for transfer of $\mathrm{H}^{+}$.

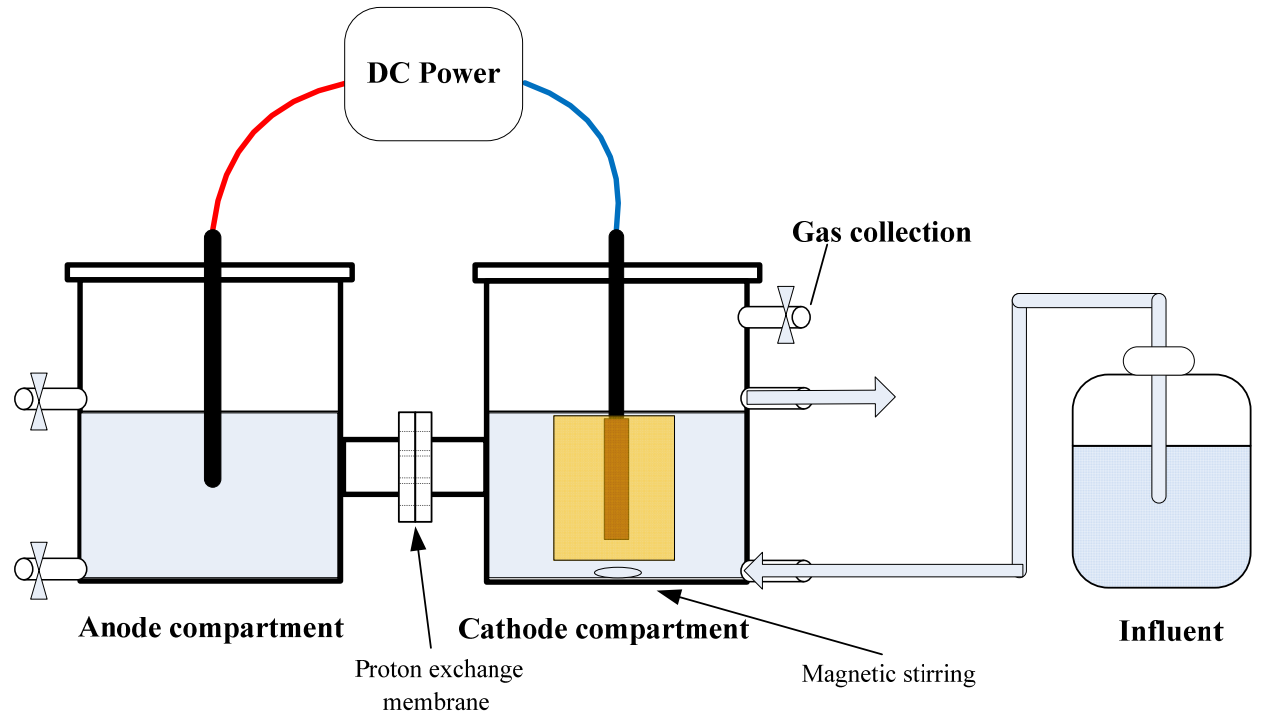

Fig. 1. The construction of the two-compartment bio-electrochemical reactor.

\subsection{Inoculum and synthetic medium}

The inoculum was taken from Liuyang River sediments (Liuyang, China) and $200 \mathrm{~mL}$ of inoculum was injected into the cathode compartment. The influent was synthesized, and an appropriate amount of $\mathrm{NH}_{4} \mathrm{HCO}_{3}$, $\mathrm{NaHCO}_{3}$ and $\mathrm{KH}_{2} \mathrm{PO}_{4}$ were added in deionized water as previous described [18]. Influent containing $5 \mathrm{mg} / \mathrm{L}$ perchlorate should be exposed to nitrogen before flowing into the reactor. The $\mathrm{pH}$ of the influent solution and the anode compartment solution were adjusted to $7.0 \pm 0.2$ with $0.5 \mathrm{M} \mathrm{H}_{2} \mathrm{SO}_{4}$.

\subsection{Biofilm sample collection and analysis}

The microbial samples attached on the activated carbon felt were collected and stored frozen at $-27^{\circ} \mathrm{C}$ to explore the microbial and species composition at the biofilms of the BER system.

Inoculum was also saved. Samples of biofilm analysis were taken in duplicate and numbered A, B. The results of the two samples were summed up for the analysis of microbial diversity. The total DNA was extracted using the E.Z.N.A. ${ }^{\circledR}$ Soil DNA Kit (Omega Bio-tek, Norcross, USA) [19]. 16S rRNA genes were amplified using the universal bacteria forward primer $338 \mathrm{~F}$ (5'ACTCCTACGGGAGGCAGCAG-3') and reverse primer 806R (5'-GGACTACHVGGGTWTCTAAT-3'), which were targeting the V3-V6 hypervariable regions. The 50$\mu \mathrm{L}$ PCR reaction mixture was composed of $5 \mu \mathrm{L} 1 \times$
FastPfu Buffer, $0.25 \mathrm{mM}$ dNTPs, $10 \mu \mathrm{M}$ each of forward and reverse primers, $0.3 \mu \mathrm{L}$ FastPfu Polymerase (Trans Gen Biotech, China), and 10 ng DNA template [17]. The PCR amplicons were sequenced on an Illumina MiSeq platform with the standard protocols by Shanghai Majorbio Technology.

\subsection{Analysis methods and calculation}

The liquid samples were collected from the system with 5 $\mathrm{mL}$ centrifuge tube, and filtered twice with a $0.22 \mu \mathrm{m}$ filter immediately. Perchlorate was analyzed by ion chromatography (Dionex ISC 900, USA) containing an AS16 column $(4 \times 250 \mathrm{~mm})$ and AG16 pre-column $(4 \times$ $50 \mathrm{~mm}$ ), and an eluent concentration of $35 \mathrm{mM} \mathrm{KOH}$. The $\mathrm{pH}$ meter (pHS-3C, Leici, China) was used to test the $\mathrm{pH}$ value.

Perchlorate surface loading $\left(\mathrm{mg} / \mathrm{m}^{2} \cdot \mathrm{d}\right)$ was calculated as followed:

$$
\mathrm{J}=\left(\mathrm{C}_{0}-\mathrm{C}\right) \mathrm{Q} / \mathrm{A}
$$

in which $C_{0}$ and $C$ are the influent and effluent perchlorate concentration $(\mathrm{mg} / \mathrm{L}), Q$ is the influent flow rate of the BER system $(\mathrm{L} / \mathrm{d})$, and $A$ is the membrane surface area $\left(\mathrm{m}^{2}\right)$.

\section{Results and discussion}

\subsection{Selection of inoculum sampling points}


The fireworks discharge area of Liuyang River sediments were used as the source of inoculated microorganisms. Liuyang city is known as the "hometown of fireworks" and the fireworks have been set off for decades, leading to a high level of perchlorate contamination in the river, sediment and soil. The average concentration of perchlorate in groundwater from these areas has reached $170 \mathrm{mg} / \mathrm{L}$. As a fireworks display platform and a fireworks display venue for holiday celebrations, the fireworks display intensity in this area was large. After discharge of firecrackers, most of particles fall into the Liuyang River and the perchlorate concentration was higher than $1.0 \mathrm{mg} / \mathrm{L}$. It can be inferred that PRB were widely distributed and had strong microbial activity. Therefore, the river sediments in this area were chosen as the source of inoculated microorganisms to degrading perchlorate.

\subsection{Inoculation of BER}

Influent containing $5 \mathrm{mg} / \mathrm{L}$ perchlorate was added to the cathode compartment, and the electrodes were not energized for the first 5 days. Because the sediments contained varies organic matters, which could be used as electron donors, and the microorganisms consumed the carbon source presented in the sludge during this period. After 5 days later, the reactor was powered on, the current intensity maintains at $10 \mathrm{~mA}$, and the influent hydraulic retention time (HRT) was set for $72 \mathrm{~h}$ for continuous flow anaerobic culture and domestication. The temperature was ambient temperature $\left(25^{\circ} \mathrm{C}-35^{\circ} \mathrm{C}\right)$, and the $\mathrm{pH}$ of influent solution was $7.0 \pm 0.2$.

The influent and effluent concentrations of perchlorate and the perchlorate removal rate are shown in Fig. 2. The first day after the reactor continuously enters the water, the excess inoculum was discharged. At the beginning stage, the perchlorate degradation rate reached about $50 \%$, and then decreased rapidly. This may be due to the adsorption of the inoculum, but with the discharge of excess inoculum and desorption, the perchlorate degradation rate was gradually reduced. When the inoculation progressed to the day 7 , the removal rate began to rise slowly. With the influent perchlorate decrease to $5 \mathrm{mg} / \mathrm{L}$, the removal rate fell to around $20 \%$ again, it may be the temperature was too high $\left(35^{\circ} \mathrm{C}\right)$ and then inhibited the activity of microorganisms. At this time, the biofilm was initially formed on the activated carbon felt. When influent perchlorate increased to $6 \mathrm{mg} / \mathrm{L}$, the removal rate had risen again and reached $78.64 \%$ on day 21 , and remained stable at above $80 \%$ for the next 4 days. According to Eq.(1), a removal flux of $178.68 \mathrm{mg} / \mathrm{m}^{2} \cdot \mathrm{d}$ was occurred when the removal rate reached $84.13 \%$. In addition, gray biofilms had been formed on the surface of activated carbon felt. This phenomenon can be considered that the inoculation phase was basically completed.

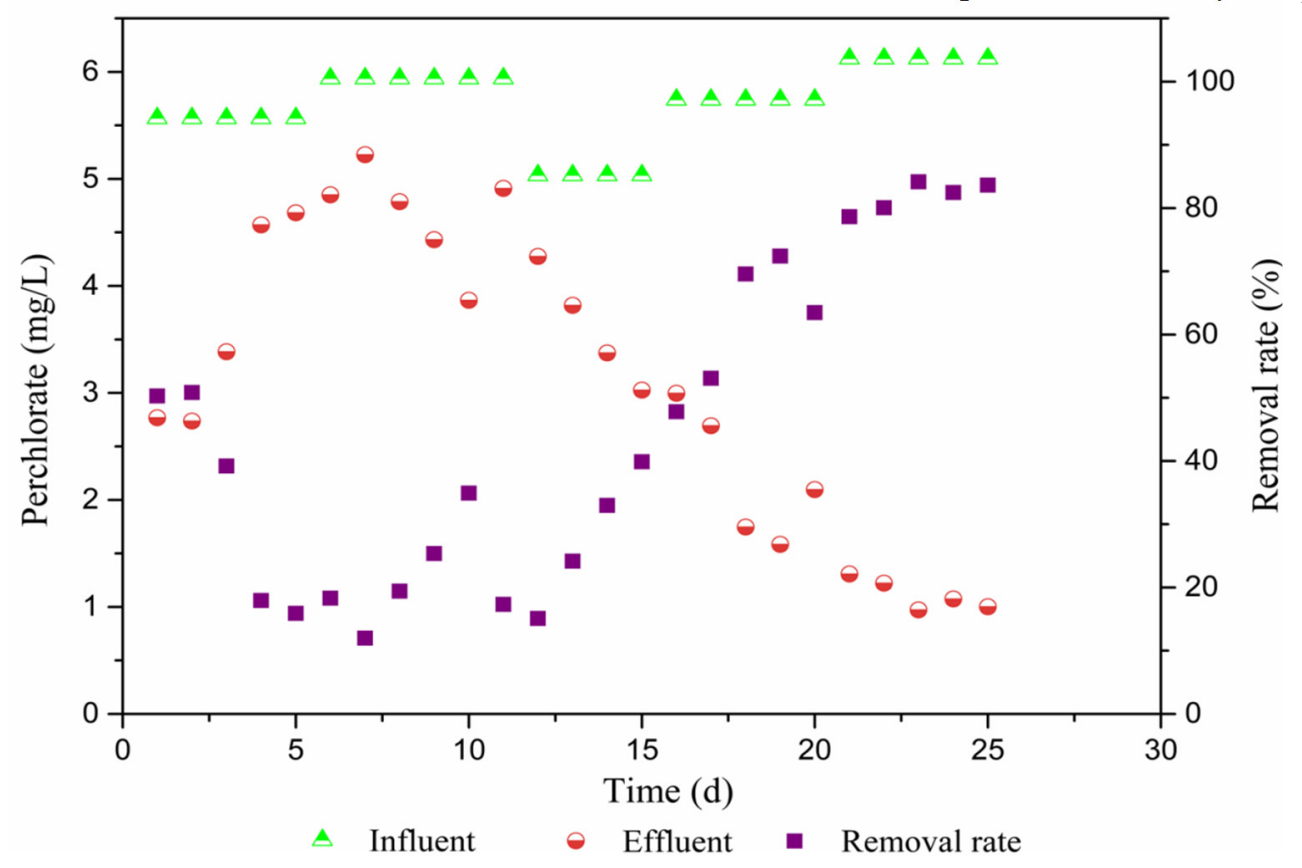

Fig. 2. Perchlorate concentrations and removal rate in the BER at different stages.

\subsection{Microbial community composition}

To confirm the biofilm composition, the high-throughput sequencing was carried out to analyze the diversity and structure of microbial community at biofilm. The effective sequence length of the three samples ranged from 46292 to 51044 , and the coverage of the sample sequencing results at the $97 \%$ similarity level was above $99 \%$, indicating that the sequencing of the three samples was reasonable, and the sequencing results could reflect the real situation of the microorganisms in the sample. Fig. 3 shows the Venn diagrams of three samples to evaluate the distribution of operational taxonomic units (OTUs) among three samples. A total of 406 OTUs were obtained based on $97 \%$ identity. The shared OTUs were 115 among 
three samples which mainly belonged to Firmicutes $(85.52 \%)$ and Bacteroidetes $(9.48 \%)$, while the Actinobacteriria and Proteobacteria were represented about $3.04 \%$ and $1.16 \%$, respectively. These results indicated that these microorganisms exist in the whole operation process. (a)

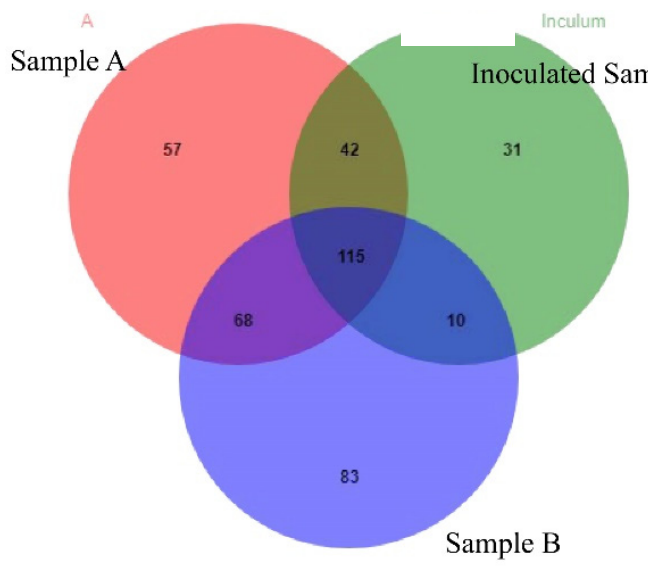

(b)

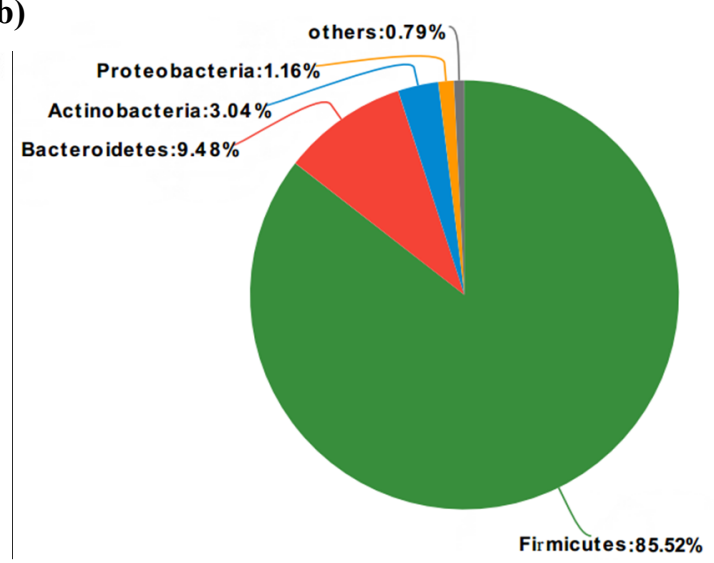

Fig. 3. (a) Venn diagrams of three samples and (b) the shared microorganisms at phylum-level.

Fig. 4 shows the microbial composition of inoculum and biofilm samples at phylum-level. The biofilm samples $\mathrm{A}$ and $\mathrm{B}$ had similar microbial composition, but the ratio was different. As shown in Fig. 4, Firmicutes was the dominate phyla and had a slight increase during inoculation phase. But Bacteroidetes accounted for a large proportion in inoculum, it was relatively small in biofilm after acclimation. Denitrifying bacteria (DB) and perchlorate-reducing bacteria (PRB) mainly belong to Proteobacteria. The growth of Proteobacteria indicated that the proportion of PRB on biofilm is increase slowly.
From the test results, it shown that the proportion of Gammaproteobacteria and Baltaproteobacteria had increased from the color of community heatmap (data were not shown). Bacilli and Clostridia belong to Firmicutes and many PRB is a branch of Firmicutes [20]. Bacilli and Clostridia were dominant at class-level both in inoculum and biofilm, with the relative abundance about $56 \%-72 \%$ and $17 \%-23 \%$, respectively. These results confirmed that the biofilm in the reactor had been successfully acclimated, and the BER system could biodegradation perchlorate effectively.

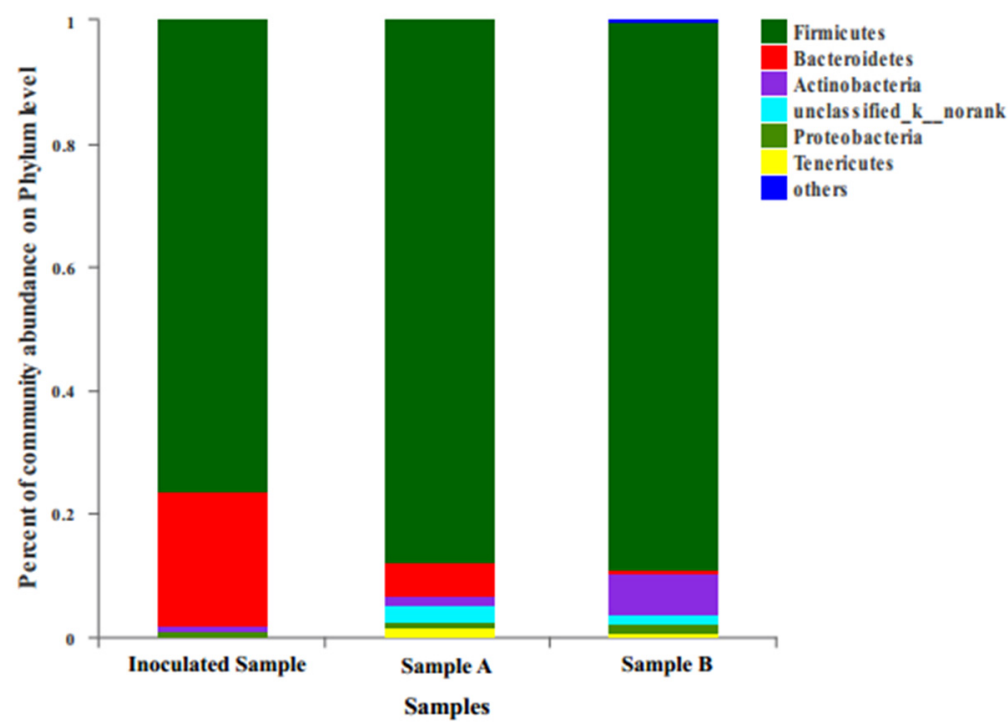

Fig. 4. Microbial communities at the phylum-level (with cut-off threshold of $99 \%$ similarity).

\section{Conclusion}

In this study, the inoculum was taken from Liuyang River sediments to inoculum in a bio-electrochemical system to bio-reduction of perchlorate. The efficient degradation of perchlorate was achieved in the system by utilizing hydrogen as the electron donor. When the current intensity was $10 \mathrm{~mA}$ and HRT was $72 \mathrm{~h}$, the removal rate of perchlorate (initial concentration was $5 \mathrm{mg} / \mathrm{L}$ ) reached $84.13 \%$ and the removal flux of $178.68 \mathrm{mg} / \mathrm{m}^{2} \cdot \mathrm{d}$ was achieved. The biofilm in the reactor had been successfully acclimated. Firmicutes and Bacteroidetes were the dominant phyla during reactor operation, Actinobacteriria, Proteobacteria, and Tenericutes also constituted a proportion in the biofilm. Bacilli and Clostridia were 
dominant at classe-level both in inoculum and biofilm, with the relative abundance about $56 \%-72 \%$ and $17 \%$ $23 \%$, respectively. These results are helpful for a systematic understanding of microbial communities in auto-hydrogenotrophic degradation process.

This research was financially supported by the project of National Natural Science Foundation of China (NSFC) (Nos. 51709104, 21806034), the Natural Science Foundation of Hunan Province (Nos. 2017JJ3148) and the Key Research and Development Program in Hunan Province (Nos.2017SK2381).

\section{Reference}

1. Y. Zhu, N. Gao, W. Chu, S. Wang, J. Xu. Chemosphere, 148, 188-194 (2016)

2. H.P. Zhao, S. Van Ginkel, Y.N. Tang, D.W. Kang, B. Rittmann, R. Krajmalnik-Brown. Environ. Sci. Technol., 45, 10155-10162 (2011)

3. W. Song, B. Gao, H. Wang, X. Xu, M. Xue, M. Zha, B. Gong. Bioresour. Technol., 240, 68-76 (2017)

4. J.K. Choe, M.H. Mehnert, J.S. Guest, T.J. Strathmann, C.J. Werth. Environ. Sci. Technol., 47, 4644-4652 (2013)

5. X. Chen, Y. Liu, L. Peng, B.J. Ni. Chem. Eng. J., 316, 82-90 (2017)

6. Y.H. Luo, R. Chen, L.L. Wen, F. Meng, Y. Zhang, C.Y. Lai, B.E. Rittmann, H.P. Zhao, P. Zheng. Environ. Sci. Technol., 49, 2341-2349 (2015)

7. B.E. Logan. Environ. Sci. Technol., 35, 482a-487a (2001)

8. H. Ma, N.A. Bonnie, M. Yu, S. Che, Q. Wang. J. Water Reuse and Desal., 6, 82-107 (2016)
9. F.B. Yao, Y. Zhong, Q. Yang, D.B. Wang, F. Chen, J.W. Zhao, T. Xie, C. Jiang, H.X. An, G.M. Zeng, X.M. Li. J. Hazard. Mater. , 323, 602-610 (2017)

10. Q. Yang, F.B. Yao, Y. Zhong, D.B. Wang, F. Chen, J. Sun, S. Hua, S.B. Li, X.M. Li, G.M. Zeng. Chem. Eng. J., 306, 1081-1091 (2016)

11. X. Xu, B. Gao, B. Jin, H. Zhen, X. Wang, M. Dai. J. Hazard. Mater., 285, 228-235 (2015)

12. Y. Wang, L. Jin, M.A. Deshusses, M.R. Matsumoto. Chem. Eng. J., 232, 388-396 (2013)

13. H.P. Zhao, A. Ontiveros-Valencia, Y. Tang, B.O. Kim, Z.E. Ilhan, R. Krajmalnik-Brown, B. Rittrnann. Environ. Sci. Technol., 47, 1565-1572 (2013)

14. Z.C. Wang, M.C. Gao, Y. Zhang, Z.L. She, Y. Ren, Z. Wang, C.C. Zhao. J. Environ. Manage., 142, 10-16 (2014)

15. X.M. Chen, Y.W. Liu, L. Peng, B.J. Ni. Chem. Eng. J., 316, 82-90 (2017)

16. M. Prosnansky, Y. Sakakibara, M. Kuroda. Water Res., 36, 4801-4810 (2002)

17. Y. Zhong, Q. Yang, G. Fu, Y. Xu, Y. Cheng, C. Chen, R. Xiang, T. Wen, X. Li, G. Zeng. J. Hazard. Mater., 342, 150-157 (2018)

18. K.F. Ettwig, T. van Alen, K.T. van de Pas-Schoonen, M.S.M. Jetten, M. Strous. Appl. Environ. Microbiol., 75, 3656-3662 (2009)

19. Y. Zhong, X. Li, Q. Yang, D. Wang, F. Yao, X. Li, J. Zhao, Q. Xu, C. Zhang, G. Zeng. J. Hazard. Mater., 307, 82-90 (2016)

20. Y. Zhu, M. Wu, N. Gao, W. Chu, S. Wang. Chemosphere, 165, 134-143 (2016) 